\title{
In utero exposure to germinated brown rice and its oryzanol-rich extract attenuated high fat diet-induced insulin resistance in F1 generation of rats
}

Hadiza Altine Adamu ${ }^{1}$, Mustapha Umar Imam ${ }^{1 *}$, Der-Jiun Ooi ${ }^{1}$, Norhaizan Mohd Esa ${ }^{2}$, Rozita Rosli ${ }^{3}$ and Maznah Ismail ${ }^{1,2^{*}}$

\begin{abstract}
Background: The development of insulin resistance is multifactorial, with maternal pre- and postnatal nutrition having significant influences. In this regard, high fat diet (HFD) feeding in pregnancy has been shown to increase risks of metabolic diseases. Thus, we investigated the effects of supplementation of HFD with germinated brown rice (GBR) and GBR-derived gamma oryzanol-rich extract (OE) on insulin resistance and its epigenetic implications in pregnant rats and their offsprings.

Methods: Pregnant female Sprague dawley rats were fed with HFD alone, HFD + GBR or HFD + OE (100 or 200 $\mathrm{mg} / \mathrm{kg} /$ day) throughout pregnancy and lactation. Their offsprings were weaned at 4 weeks post-delivery and were followed up until 8 weeks. Serum levels of adipokines were measured in dams and their offsprings, and global DNA methylation and histone acetylation patterns were estimated from the liver.

Results: The dams and offsprings of the GBR and OE groups had lower weight gain, glycemic response, 8-IsO prostaglandin, retinol binding protein 4 and fasting insulin, and elevated adiponectin levels compared with the HFD group. Fasting leptin levels were lower only in the GBR groups. Hepatic global DNA methylation was lower in the GBR groups while hepatic $\mathrm{H} 4$ acetylation was lower in both GBR and OE dams. In the offsprings, DNA methylation and $\mathrm{H} 4$ acetylation were only lower in the OE group. However, dams and offsprings of the GBR and OE groups had higher hepatic $\mathrm{H3}$ acetylation.
\end{abstract}

Conclusions: GBR and OE can be used as functional ingredients for the amelioration of HFD-induced epigeneticallymediated insulin resistance.

Keywords: DNA methylation, Epigenetics, Germinated brown rice, Gamma oryzanol, Histone acetylation, Insulin resistance, Nutrigenomics

\section{Background}

The prevalence of metabolic syndrome, characterized by hypertension, abdominal obesity, T2D, dyslipidemia and insulin resistance, is on the rise. Its etiology is complex with both environmental and genetic contributions [1]. Diet is an important environmental factor that could influence the risk of insulin sensitivity, and in fact,

\footnotetext{
*Correspondence: mustyimam@gmail.com; maznahis@upm.edu.my ${ }^{1}$ Laboratory of Molecular Biomedicine, Institute of Bioscience, Universiti Putra Malaysia, 43400 UPM Serdang, Selangor, Malaysia

Full list of author information is available at the end of the article
}

maternal pre- and post-natal nutrition has been demonstrated to modulate risk of insulin resistance later in life [2]. Furthermore, the preference of diets that cause increased visceral fat deposition and elevated rates of lipolysis has been linked with significant rise in metabolic diseases such as insulin resistance [3]. Accordingly, maternal nutrition that promotes increased fat deposition has been shown to promote the risk of insulin resistance in offsprings especially when consumed during pregnancy. Similarly, from a nutritional point of view, bioactive compounds are able to change global 
DNA methylation, with implications on functional activation or repression of genes [4]. A few bioactives are specific to certain cereals such as gamma oryzanol in rice [5], which is a mix of ferulic acid esters with phytosterols [6]. It is reported to have various functional properties including cholesterol-lowering effects and antioxidative properties [7]. It was also reported to be effective in reducing weight [8], which is an important determinant of metabolic status. Moreover, excessive weight can lead to various metabolic perturbations with epigenetic implications on the risk of metabolic disease in offsprings [9].

Germinated brown rice (GBR) has higher amounts of bioactive compounds than brown rice, which confers superior bioactivity including antiobesity and antidiabetic effects [10]. In view of the current epidemic of diabetes in children and the contributions of nutrition on metabolic disease outcomes in offsprings, we hypothesized that the reported bioactivities of GBR and oryzanol may be beneficial in ameliorating high fat diet- (HFD-) induced metabolic perturbations. Moreover, we have demonstrated that GBR can ameliorate the risk of metabolic diseases, and that oryzanol is one of the important bioactives in GBR that contributes to its bioactivities [10]. Thus, in the present study, GBR and GBR-derived gamma oryzanol-rich extract were evaluated for their ability to attenuate metabolic disease induced in F1 offsprings of rats that consumed HFD during pregnancy.

\section{Methods}

\section{Chemicals, enzyme-linked immunosorbent assays and epigentic kits}

All solvents were of analytical grade from Merck. Biotechnolgy grade water (7732-18-5) and commercial oryzanol were obtained from Sigma-Aldrich. Rat adiponectin (Catalog No EZRADP-62 K) and insulin (Catalog No EZRMI-13 K) enzyme-linked immunosorbent assay (ELISA) kits were procured from Millipore Corporation. Rat retinol binding protein-4 dual ELISA kit (Catalog No AG-45A-0012YEK-K101) was obtained from Adipogen International, while 8-iso prostaglandin (Catlog No E-EL-R2488) and leptin (Catlog No E-EL-R0582) ELISA kits were purchased from Elabscience Biotechnology Co., Ltd. MethylFlash ${ }^{\mathrm{Tm}}$ Methylated DNA Quantification Kit (Fluorometric) (Catalog No P-1035), Epiquick ${ }^{\text {TM }}$ Total $^{-}$ Histone Extraction Kit (Catalog No OP-0006), Epiquick ${ }^{\text {тм }}$ Epiquick $^{\mathrm{TM}}$ Total Histone H3 Acetylation Detection Fast Kit (Fluorometric) (Catalog No P-4031) and Epiquick ${ }^{\text {тм }}$ Total Histone H4 Acetylation Detection Fast Kit (Fluorometric) (Catalog No P-4033) were obtained from EPIGENETIK.

\section{Germination of brown rice and extraction of oryzanol}

Brown rice (MR220 variety) was obtained from PadiBeras Nasional Berhad (BERNAS) (Selangor, Malaysia), and was germinated as described previously [11]. Briefly, $500 \mathrm{~g}$ of brown rice was washed twice using tap water, after which sodium hypochlorite was added at a ratio of 1:2 (w/v) and left to soak for $30 \mathrm{~min}$. It was then drained and rinsed with distilled water and soaked in hydrogen peroxide (H2O2) 1:2 (w/v) and incubated (Glass door low temperature (BOD) incubators SD-450) for $6 \mathrm{~h}$ at $37{ }^{\circ} \mathrm{C}$. Then, $\mathrm{H}_{2} \mathrm{O}_{2}$ was drained and the rice was incubated once again in closed plastic container for $18 \mathrm{~h}$ at $37{ }^{\circ} \mathrm{C}$ and later oven-dried (Memmert) at $50{ }^{\circ} \mathrm{C}$ until moisture content of $8-11 \%$ was achieved using the oven dry method. We have previously demonstrated that the MR220 variety, which is commonly consumed in Malaysia, had polyhedral granular starch particles that measured around 2 to $8 \mu \mathrm{m}$ for all starches. Its amylose content was generally less than $30 \%$, which was significantly lowered along with its gelatinization and pasting properties after germination [12]. In this study, the GBR was ground with a stainless steel grinder (Waring Commercial) and used in preparing the rat pellets.

The extraction of gamma oryzanol from GBR was done according to Zamri [6]. Hexane (400 ml; 70\%) was mixed with approximately ground GBR (100 g) and left to soak for $30 \mathrm{~min}$. It was then centrifuged for $20 \mathrm{~min}$ at 34,800 g. Whattman No. 1 filter paper with the aid of a glass funnel was used to filter the supernatant and the extraction process repeated two more times for a total of 3 extractions. The filtered supernatants were pooled and dried using a rotary evaporator (Rotavapor ${ }^{\circledR}$ R-210, BUCHI). The oryzanol yields were reported in our earlier publication as 30.38-64.22 mg/g GBR [13].

\section{Experimental design}

All animal experiments were implemented in accordance with the guidelines for the use of animals as approved by the Animal Care and Use Committee, Faculty of Medicine and Health Sciences, Universiti Putra Malaysia (approval number UPM/FPSK/PADS/BR-UUH/00360). Fifteen female old Sprague Dawley rats aged 8 weeks were raised on regular chow diet by the breeder were fed regular chow ad libitum and free water access during the acclimatization week. The animal house was wellventilated with a $12 \mathrm{~h}$ light/dark cycle at the ambient temperature of $25-30{ }^{\circ} \mathrm{C}$, throughout the experimental period. The animals were then grouped $(n=3)$ into high fat diet (HFD), regular chow (N), high fat diet with $50 \%$ GBR (GBR), high fat diet with high dose oryzanol extract (200 mg/kg body weight, OEHD) and high fat diet with low dose oryzanol extract $(100 \mathrm{mg} / \mathrm{kg}$ body weight, OELD). Oryzanol at 100 and $200 \mathrm{mg} / \mathrm{kg}$ body weights were safe and produced metabolic changes in our earlier publications [10], hence our choice of these concentrations in the present study. Male Sprague Dawley rats that had been fed with regular chow were mated with 
the female rats. The female rats were maintained on these diets during pregnancy and lactation. Oryzanol extract (high and low dose) were administered via intragastric gavage. The high Fat Diet (HFD) formulation was made up of $47.7 \%$ total carbohydrate, $16.1 \%$ protein, $31.1 \%$ fat, $2.5 \%$ fiber and $5.1 \%$ mineral and vitamin and prepared using $50 \%$ normal rat chow powder, $24 \%$ Mazola oil, 20\% Nespray full-cream milk powder, 6\% sugar and $50 \mathrm{~g}$ of starch, placed in an oven at $60{ }^{\circ} \mathrm{C}$ for $24 \mathrm{~h}$, cut into smaller pieces and then used to feed the rats. The caloric compositions of the pellets given to each group are shown in Table 1. Food intake was set at $30 \mathrm{kcal} / 100 \mathrm{~g}$ body weight/day for all the groups (Table 1). Accordingly, the weights of the rats were measured weekly, and the total amount of feed given daily (grams) was reviewed every week based on the new weights of the rats. After delivery, male offsprings were chosen for follow up on the effects of the perinatal interventions on insulin resistance markers. At 4 weeks postdelivery, the male offsprings ( $n=6$ per group) were weaned and maintained on regular chow $(30 \mathrm{kcal} / 100 \mathrm{~g}$ body weight/day) for another 4 weeks. Energy efficiency of body weight (EE-BW) was calculated for the offsprings as weight gained in each group in g divided by energy intake in kJ [14]. Dams were sacrificed via when they were approximately 16 weeks old, and after weaning off the offsprings. Weekly weights of the offsprings were taken for the 8 weeks, after which they were sacrificed. Prior to sacrifice, the animals were fasted overnight and subsequently dissected after light anesthesia to harvest the liver. Blood $(10 \mathrm{~mL} / \mathrm{rat})$ was collected via cardiac puncture before dissection and centrifuged at $3000 \mathrm{rpm}$ for $10 \mathrm{~min}$ at $4{ }^{\circ} \mathrm{C}$ to separate the serum. The liver was removed immediately, washed with ice-cold saline, dried with filtered paper, and then stored in RCL2 ${ }^{\circ}$ Solution (ALPHELYS, France) at $-80{ }^{\circ} \mathrm{C}$.

\section{Oral glucose tolerance test}

In order to appraise the effects of the different treatments in the Dams and offsprings on systemic glucose

Table 1 Animal groups and diets

\begin{tabular}{|c|c|c|c|}
\hline Groups & $\begin{array}{l}\text { High fat } \\
\text { diet }^{\mathrm{a}}(\%)\end{array}$ & Others & $\begin{array}{l}\text { Dietary energy } \\
\text { contributions }{ }^{a} \\
\text { (Kcal/100 g pellet) }\end{array}$ \\
\hline Normal & 0 & $100 \%$ normal rat pellet & 335 \\
\hline High fat diet & 50 & $50 \%$ normal rat pellet & 548 \\
\hline $\begin{array}{l}50 \% \text { Germinated } \\
\text { brown rice }\end{array}$ & 50 & $\begin{array}{l}50 \% \text { germinated } \\
\text { brown rice }\end{array}$ & 554 \\
\hline Low dose OE & 100 & 100 mg/kg/day OE & 554 \\
\hline High dose OE & 100 & 200 mg/kg/day OE & 554 \\
\hline
\end{tabular}

$O E$ oryzanol extract. ${ }^{a}$ Energy contributions are as reported in our earlier study (reference [13]) homeostasis, oral glucose tolerance test (OGTT) was performed. Rats were initially fasted overnight prior to OGTT via intra-gastric gavage using a glucose solution ( $2 \mathrm{~g} / \mathrm{kg}$ body weight). This was done after weaning in the dams and at 8 weeks post-delivery for the offsprings. Blood samples were taken from the tail vein at $0,30,60$, 90 and 120 min using a glucometer (Accu-Chek, Roche Diagnostics).

\section{Serum insulin, adiponectin, leptin, RBP 4 and 8-iso prostaglandin}

Serum levels of these markers were determined using the respective ELISA kits according to the manufacturers' instructions. Absorbance were read on a micro plate reader at the recommended wavelengths and the results calculated from the respective standard curves; Insulin $(y=$ $\left.0.153 \mathrm{x}+0.3572, R^{2}=0.9911\right)$, Adiponectin $(\mathrm{y}=0.0026 \mathrm{x}+$ $\left.0.1455, R^{2}=0.9985\right)$, Leptin $\left(\mathrm{y}=.2215 \mathrm{x}+0.0286, R^{2}=\right.$ $0.9982)$, RBP $4\left(\mathrm{y}=0.1041 \mathrm{x}+0.2977, R^{2}=0.9875\right)$ and 8 -iso prostaglandin $\left(\mathrm{y}=0.0024 \mathrm{x}+0.3167, R^{2}=0.9781\right)$.

\section{DNA isolation}

DNA was extracted using the ZR-Duet ${ }^{\mathrm{Tm}}$ DNA/RNA MiniPrep kit (Zymo Research) according to the manufacturer's instructions. Then, the quality and quantity assessments of the extracted samples were done using Implem NanoPhotometer ${ }^{\circ}$ prior to storage at $-80^{\circ} \mathrm{C}$.

\section{Methylated DNA quantification (fluorometric)}

Methylated DNA was quantified using a fluorometric kit according to the manufacturers' instructions. Absorbance was read on a micro plate reader at the recommended wavelength and the result expressed as fold change with respect to the $\mathrm{N}$ group.

\section{Total histone extraction}

Extraction of total histone was done according to the manufacturers' instructions. Quantification of protein was calculated using BSA as standard curve $(\mathrm{y}=2.3639 \mathrm{x}$ $\left.0.0234, R^{2}=0.9981\right)$.

\section{Total histone $\mathrm{H} 3$ and $\mathrm{H} 4$ acetylation}

Total Histone $\mathrm{H} 3$ and $\mathrm{H} 4$ Acetylation were performed according to the manufacturers' instructions. Absorbance were read on a micro plate reader at the recommended wavelength and the respective standard curves calculated $\left(\mathrm{y}=8.0054 \mathrm{x}+411.8, \quad R^{2}=0.9618\right)$ and $(\mathrm{y}=$ $\left.835.06 \mathrm{x}+407.6, R^{2}=0.9978\right)$. The amount of acetyl histone $\mathrm{H} 3$ and $\mathrm{H} 4$ were then determined by the formula below:

$$
\text { Amount }(\mathrm{ng} / \mathrm{mg} \text { protein })=\frac{\mathrm{RFU}(\text { sample }- \text { blank })}{\text { Protein }(\mathrm{ug}) * \times \text { slope }} \times 1000
$$

* Histone extract amount added into the sample well. 


\section{Statistical analysis}

Data were expressed as mean \pm standard deviation, and the means were compared using ANOVA (analysis of variance) with Tukey's multiple comparison test.

\section{Results}

\section{Body weight}

The body weights (Fig. 1) of the offsprings increased over the course of 8 weeks, with similar EE-BW for all the groups (Table 2). A significant difference $(P=0.00001, F=$ 63.88) was observed between the HFD and treatment groups at week 8 with a percentage change of $25,42,34$ and $43.5 \%$ in the N, GBR, OEHD and OELD respectively. Treatment groups were observed to weigh less than the $\mathrm{N}$ group in the first few weeks.

\section{Oral glucose tolerance test}

Blood glucose levels in HFD dams (Fig. 2a) after oral administration of glucose solution $(2 \mathrm{~g} / \mathrm{kg}$ body weight) were highest at $30 \mathrm{~min}$. A significant difference between HFD and the treatment groups was observed at $0(P=0.00001, F=25.99), 60 \quad(P=0.0002$, $F=16.54), 90(P=0.001, F=11.4)$ and $120 \mathrm{~min}(P=$ $0.00001, F=54.46)$ respectively. In the offsprings (Fig. 2b), all the groups peaked at $30 \mathrm{~min}$ after oral administration except the HFD group, which peaked at $60 \mathrm{~min}(P=0.001, F=6.44)$ and was significantly higher than the rest of the groups at $90(P=0.00001$, $F=71.3)$ and $120 \mathrm{~min}(P=0.00001, F=38.23)$.

\section{Serum insulin}

The HFD group had the highest fasting serum insulin level among the dams (Fig. 2c). The GBR and OEHD groups had significantly different levels compared to HFD $(P=0.02, F=7.79)$ and $\mathrm{N}(P=0.01, \quad F=8.65)$ groups. In the offsprings (Fig. 2d) fasting serum insulin levels in all the treatment groups differed significantly $(P=0.0001, F=36.69)$ compared to HFD group except OELD group. HFD group had the highest serum insulin, as was the case in the dams.

\section{Adiponectin}

In the dams (Fig. 3a), serum adiponectin levels were highest in the GBR group, while the HFD group had the lowest $(P=0.00001, F=216.18)$. The level was also lowest in the HFD offsprings in comparison with other groups, while the OEHD group had the highest level $(P=0.00001, F=49)$ (Fig. 3b).

\section{Leptin}

Leptin level in serum of dams (Fig. 3c) was lowest in the GBR group and highest in the HFD group $(P=$ $0.017, F=5.12)$. A similar trend was observed for the offsprings $(P=0.016, F=5.15)$ (Fig. 3d).

\section{Retinol binding protein 4 (RBP4)}

Serum RBP4 levels in dams (Fig. 3e) were high in the HFD and OELD groups, while they were lower in the $\mathrm{N}, \mathrm{GBR}$ and OEHD groups compared with the former groups $(P=0.00001, F=31.97)$. A similar pattern was

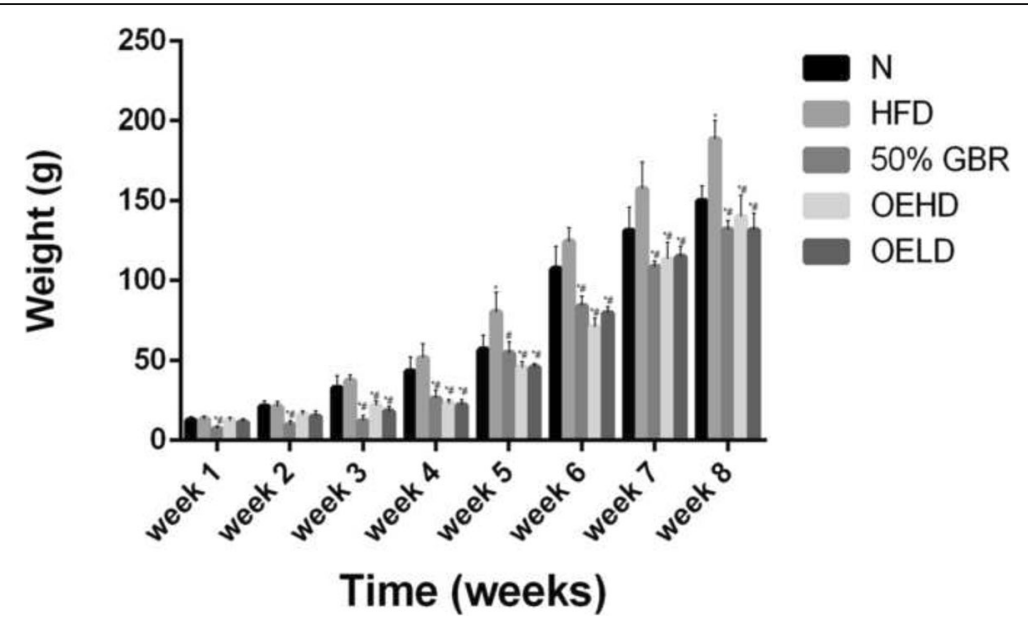

Fig. 1 A. Average weight gains of offsprings (mean $\pm S D, n=6$ ) over 8 weeks. Dams were fed the respective diets for each group, while their offsprings were fed regular rat chow after weaning and observed until 8 weeks post-delivery. Abbreviations refer to the diets given to the dams: high fat diet (HFD), normal (N), high fat diet with $50 \%$ Germinated Brown Rice (GBR), high fat diet with oryzanol-rich extract (gavage) $200 \mathrm{mg} / \mathrm{kg}$ body weight (high dose) (OEHD), high fat diet with oryzanol-rich extract (gavage) $100 \mathrm{mg} / \mathrm{kg}$ body weight (low dose)(OELD). Bars representing the same week with ${ }^{*}$ indicates statistical difference in comparison with HFD group $(p<0.05)$, \# indicates statistical difference in comparison with $N$ group $(p<0.05)$ 
Table 2 Offsprings' energy efficiency of body weight

\begin{tabular}{lllc}
\hline Groups & $\begin{array}{l}\text { Total weight } \\
\text { gained }(\mathrm{g})\end{array}$ & $\begin{array}{l}\text { Total food } \\
\text { consumed }(\mathrm{Kj})\end{array}$ & $\begin{array}{l}\text { Energy efficiency } \\
(\mathrm{g} / \mathrm{Kj})\end{array}$ \\
\hline Normal & $137.44 \pm 7.14$ & $698.45 \pm 11.55$ & $0.20 \pm 0.06$ \\
High fat diet & $175.50 \pm 9.48$ & $847.05 \pm 14.10$ & $0.21 \pm 0.07$ \\
$\begin{array}{l}50 \% \text { Germinated } \\
\text { brown rice }\end{array}$ & $124.71 \pm 3.78$ & $546.01 \pm 6.95$ & $0.23 \pm 0.05$ \\
Low dose OE & $127.80 \pm 12.26$ & $557.18 \pm 16.70$ & $0.23 \pm 0.07$ \\
High dose OE & $120.50 \pm 9.90$ & $551.24 \pm 13.08$ & $0.22 \pm 0.08$ \\
\hline
\end{tabular}

$O E$ oryzanol extract

observed in the offsprings $(P=0.00001, \quad F=178.75)$ (Fig. 3f).

\section{8 - Iso prostaglandin}

The levels of 8 - Iso prostaglandin in the $\mathrm{N}$ and treatment groups in the dams $(P=0.0008, F=11.83)$ (Fig. 4a) and offsprings ( $P=0.006, F=7.07)$ (Fig. $4 \mathrm{~b})$ were significantly lower than in the HFD group.

\section{Methylated DNA quantification}

The degree of DNA methylation was expressed in fold change with respect to the $\mathrm{N}$ group in both dams and offsprings. In the dams (Fig. 4c), the GBR group had significantly lower levels of DNA methylation compared to the HFD group. The OEHD and OELD groups had higher but statistically nonsignificant levels compared with the HFD group $(P=$ 0.0017, $F=9.82$ ). In the offsprings (Fig. 4d), however, the GBR, OEHD and OELD groups had lower DNA methylation levels compared with the HFD group $(P=$ $0.0006, F=13.05)$.

\section{Total histone $\mathrm{H} 3$ acetylation}

In the dams (Fig. 5a), total histone $\mathrm{H} 3$ acetylation (ng/mg protein) levels in the OEHD group were significantly higher than in the HFD group $(P=0.00001$, $F=23.56)$. The same pattern was observed for the offsprings $(P=0.00001, F=134.45)$ (Fig. $5 \mathrm{~b})$.

\section{Total histone $\mathrm{H} 4$ acetylation}

All the treatment groups in dams (Fig. 5c) had lower degree of $\mathrm{H} 4$ acetylation compared to the HFD group $(P=$ $0.00001, F=44.13$ ) while in the offsprings (Fig. $5 \mathrm{~d}$ ), only the $\mathrm{N}$ and OEHD groups had lower $\mathrm{H} 4$ acetylation compared to the HFD group $(P=0.00001, F=268.27)$.

\section{Discussion}

The occurrence of type 2 diabetes amongst the pediatric population is assuming pandemic proportions, and has been linked with the growing burden of obesity, which disturbs the equilibrium between insulin secretion and sensitivity leading up to insulin resistance [15]. The cause of this pandemic is multifactorial, with several studies indicating that developmental and early life environmental conditions are important variables [16]. Insulin resistance is linked to an increase in lipid accumulation around the liver and in the visceral chambers, often as a result of increased weight gain, and has been shown to correlate with lower levels of adiponectin [17].
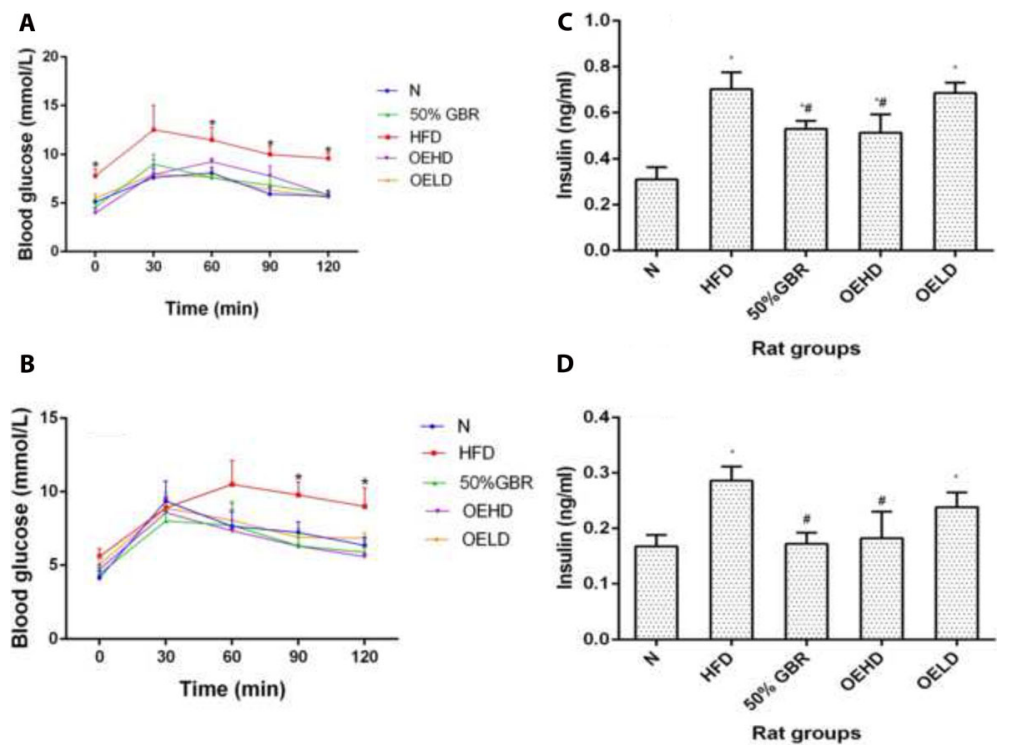

Fig. 2 OGTT profiles of a dams (mean $\pm S D, n=3$ ) and $\mathbf{b}$ offsprings (mean $\pm S D, n=6$ ), serum insulin levels of dams (c) and offsprings (d). Data for dams and offsprings represent mean $\pm \mathrm{SD}(n=3)$ and mean $\pm \mathrm{SD}(n=6)$, respectively. ${ }^{*}$ Represents significant difference between HFD and treatment groups in dams and offsprings. Bars with ${ }^{*}$ indicates statistical difference in comparison with HFD group ( $\left.p<0.05\right)$, \# indicates statistical difference in comparison with $N$ group $(p<0.05)$. Groupings are the same as Fig. 1 

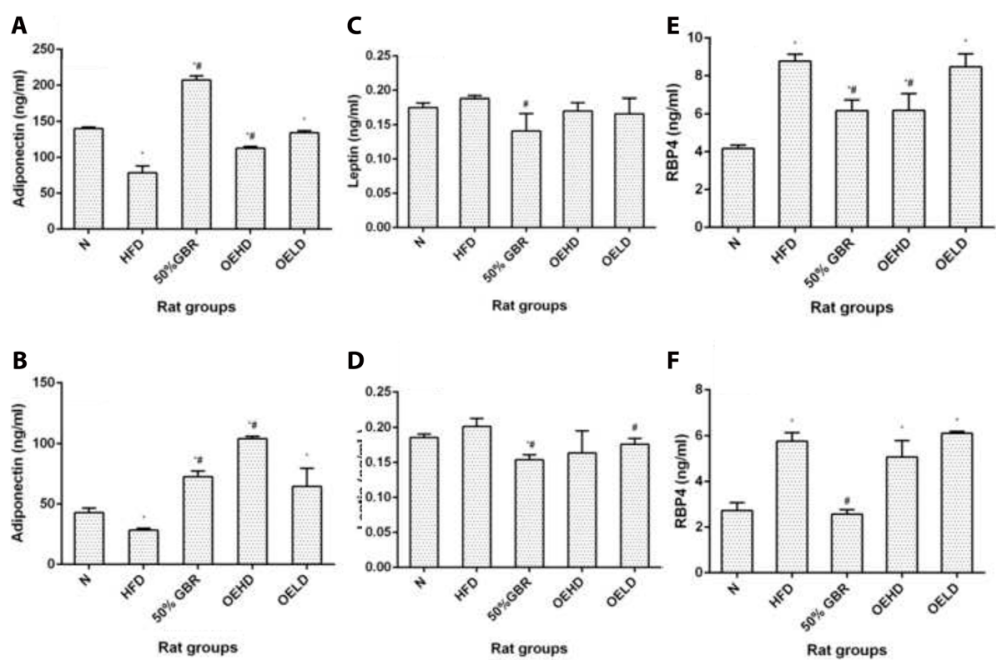

Fig. 3 Serum adiponectin levels in dams (a) and offsprings (b), and serum leptin levels in dams (c) and offsprings (d), serum retinol binding protein 4 (RBP4) levels of dams (e) and offsprings ( $\mathbf{f}$. Data for dams and offsprings represent mean \pm SD $(n=3)$ and mean \pm SD $(n=6)$, respectively. Bars with ${ }^{*}$ indicates statistical difference in comparison with HFD group $(p<0.05)$, ${ }^{*}$ indicates statistical difference in comparison with $N$ group $(p<0.05)$. Groupings are the same as Fig. 1

In the present study, the GBR groups (dams and offsprings) had low insulin levels with correspondingly high adiponectin levels. Thus, despite the low birth weight observed in GBR and OE groups the offsprings were not at risk of T2D as suggested by the raised adiponectin levels, in favour of improved insulin sensitivity.
Additionally, elevated adiponectin levels can promote insulin sensitivity via increasing fat oxidation leading to a reduction of intracellular triglyceride and fatty acid levels in muscle and liver, and overall body weight, similar to what we observed in the present study [18]. As such the enhancements in metabolic indices observed in this
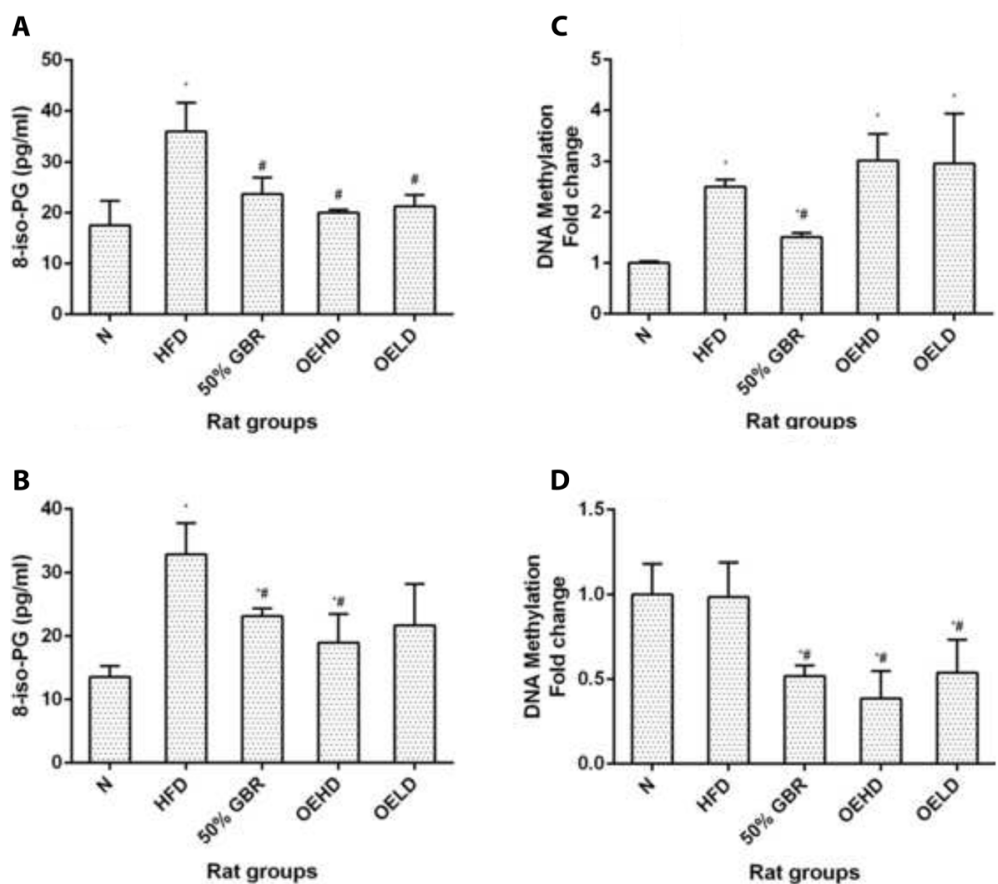

Fig. 4 Serum 8-isoprostaglandin (8-iso-PG) levels of dams (a) and offsprings (b), and fold changes in hepatic global DNA methylation in dams (c) and offsprings (d). Data for dams and offsprings represent mean \pm SD $(n=3)$ and mean $\pm \operatorname{SD}(n=6)$, respectively. Bars with ${ }^{*}$ indicates statistical difference in comparison with HFD group $(p<0.05)$, " indicates statistical difference in comparison with N group $(p<0.05)$. Groupings are the same as Fig. 1 

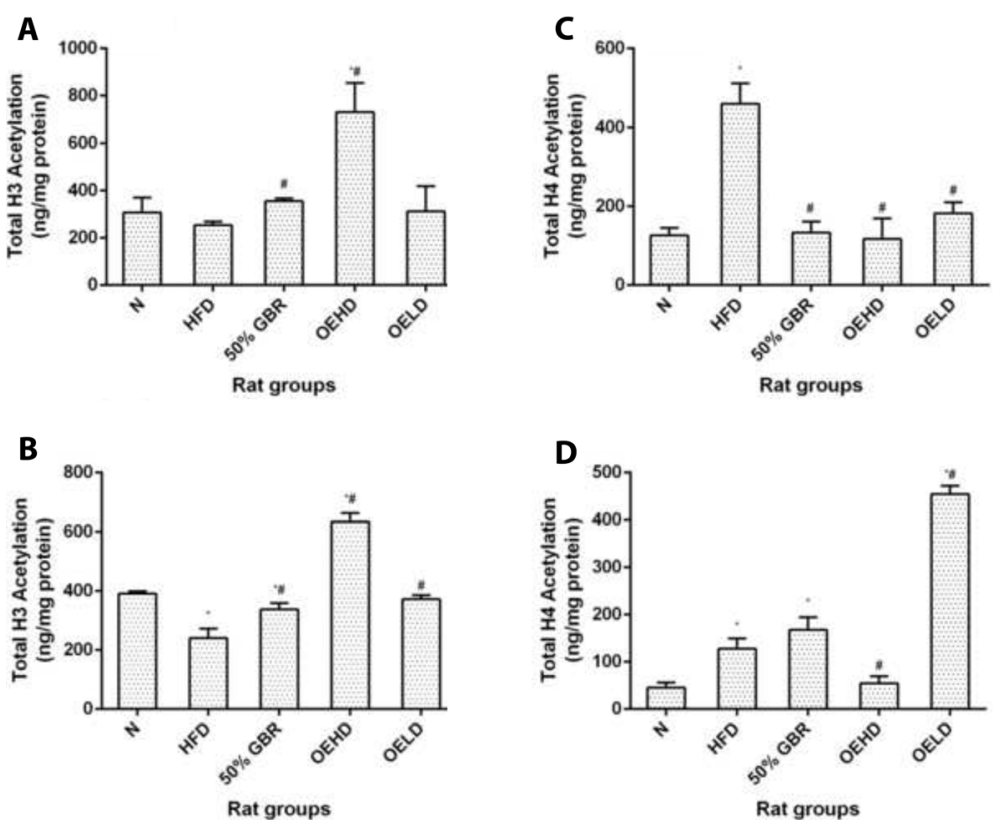

Fig. 5 Hepatic total H3 histone acetylation in dams (a) and offsprings (b), and hepatic total H4 histone acetylation in dams (c) and offsprings (d). Data for dams and offsprings represent mean \pm SD $(n=3)$ and mean \pm SD $(n=6)$, respectively. Bars with ${ }^{*}$ indicates statistical difference in comparison with HFD group $(p<0.05)$, ${ }^{*}$ indicates statistical difference in comparison with N group $(p<0.05)$. Groupings are the same as Fig. 1

study could have been due to elevated adiponectin, in addition to the lower body weights, in both dams and offsprings that consumed GBR and GBR-derived oryzanol. The lower body weights in the offsprings in this study may not have resulted from adverse intrauterine conditions, hence the correspondingly enhanced metabolic conditions. Moreover, we have reported severally that GBR could regulate weight gain and promote weight loss [10], which in the present study may have contributed towards enhanced metabolic outcomes.

As can be recalled, GBR and oryzanol produced metabolic outcomes that favoured insulin sensitivity better than HFD feeding in the dams and offsprings. The dietary fiber present in GBR can lower glycemic index via regulating intestinal glucose absorption, while other bioactives also contribute to better glycemic control through different mechanisms [10, 19]. Oryzanol was also observed to improve glycemic control in mice fed with HFD [20]. Moreover, the metabolic outcomes due to GBR and oryzanol could also have been secondary to lowered leptin levels [21]. Furthermore, there is an inverse correlation in type 2 diabetes between serum adiponectin and 8 - Iso prostaglandin, an important marker of oxidative stress [22], in line with the results we obtained for the HFD dams and offsprings whereby adiponectin levels were low and those of 8 - Iso prostaglandin were raised. Our results ware further supported by the RBP4 levels in the treatment groups, which showed patterns consistent with improved metabolic outcomes [23]. Overall, GBR and oryzanol were able to attenuate the HFD-induced metabolic perturbations in the dams and offsprings, although GBR showed better results suggesting that the presence of multiple compounds conferred superior bioactivity.

In recent years, epigenetic regulation of gene expression has surfaced as a key contributor in the development of metabolic disorders such that unfavorable conditions during the developmental phase (in-utero or lactation) could change the developmental programming of the offspring. In line with this, fetal nutrition is now acknowledged as a crucial regulator of fetal well-being affecting developmental programming $[2,4]$. The most studied epigenetic mechanisms include DNA methylation and histone modification with the former involving the methylation at 5 ' position of cytosine inside a $\mathrm{CpG}$ dinucleotide located at the promoter regions of genes, referred to as $\mathrm{CpG}$ islands. Transcriptional repression and activation in these islands is linked to hyper- and hypo-methylation, respectively [4, 24]. Our results showed that a diet of GBR in dams attenuated HFDinduced hepatic global DNA methylation changes and this in turn epigenetically influenced the lower global DNA methylation observed in their offsprings in comparison with the HFD offsprings. Post translational modifications such as acetylation of $\mathrm{H} 3$ and $\mathrm{H} 4$ also have profound effects on functional activation or repression of genes [24]. Our results also showed that GBR and GBR-derived oryzanol extract modulated $\mathrm{H} 3$ and $\mathrm{H} 4$ acetylation patterns in dams and their offsprings. 
We observed in general a trend in GBR dams, which suggested epigenetically-mediated effects on the offsprings. This trend was less apparent for oryzanol treatment. From our result, it seems plausible that the synergistic effects of the multiple bioactives in GBR on the dams targeting different metabolic pathways were responsible for the attenuation of the HFD-induced metabolic effects in the offsprings, independent of any influences on the EE-BW of the offsprings' diet. This was also the possible reason why the offsprings' of the GBR group had better outcomes compared to those of the oryzanol group, although solvent remnants may have contributed some adverse effects to the oryzanol treatment. Similarly, the influence of GBR and oryzanol on gut microbiota of the rats during pregnancy may have contributed to the differential outcomes [2]. Thus, consumption of GBR and oryzanol can be a way to modulate the risk of insulin resistance during pregnancy, the effects of which can be observed in the F1 generation.

\section{Conclusion}

In aggregate, we demonstrated the effects of GBR and oryzanol on HFD-induced insulin resistance and their epigenetic implications in F1 generation of rats. The anti-diabetic effects of GBR and oryzanol have been demonstrated previously, but this is the first report on the epigenetic implications of GBR and oryzanol supplementation in pregnant rats. Our results suggest that GBR and oryzanol can be important functional ingredients for the amelioration of HFD-induced epigeneticallymediated insulin resistance, which may have profound implications on the burden of insulin resistance in humans. These results are worth studying further in the quest for solutions for the raging obesity pandemic.

\section{Abbreviations}

BSA: Bovine serum albumin; GBR: Germinated brown rice; HFD: High fat diet; N: Normal, fed regular chow; OE: GBR-derived gamma oryzanol-rich extract; OEHD: GBR-derived gamma oryzanol-rich extract high dose; OELD: GBRderived gamma oryzanol-rich extract low dose; OGTT: Oral glucose tolerance test; RBP4: Retinol binding protein; RFU: Relative fluorescence units

\section{Acknowledgement \\ The author are grateful to PadiBeras Nasional Berhad (BERNAS) and Universit Putra Malaysia for funding this project. The authors thank the staff of the Laboratory of Molecular Biomedicine for their assistance with this study. \\ Funding \\ This project was funded by PadiBeras Nasional Berhad (BERNAS) and Universiti Putra Malaysia (vote no 63536).}

\section{Availability of data and materials}

The data that support the findings of this study are available from the corresponding authors on request.

\section{Authors' contribution}

Conception of idea and research design: HAA, MUI, MI. Conduct of research and experimentation: HAA, MUI, DJO. Data analyses: HAA, MUI, DJO. Drafting of manuscript: HAA, MUI. Review and approval of final manuscript: MUI, MI, NME, RR. All authors read and approved the final manuscript.

\section{Competing interest}

The authors declare that they have no competing interests.

\section{Consent for publication}

Not applicable.

\section{Ethics approval and consent to participate}

Ethical approval was obtained from the institutional animal care and use committee (IACUC), Faculty of Medicine and Health Sciences, Universiti Putra Malaysia.

\section{Author details}

${ }^{1}$ Laboratory of Molecular Biomedicine, Institute of Bioscience, Universiti Putra Malaysia, 43400 UPM Serdang, Selangor, Malaysia. ${ }^{2}$ Department of Nutrition and Dietetics, Universiti Putra Malaysia, 43400 UPM Serdang, Selangor, Malaysia. ${ }^{3}$ UPM-MAKNA Cancer Research Laboratory, Institute of Bioscience, Universiti Putra Malaysia, 43400 UPM Serdang, Selangor, Malaysia.

Received: 30 September 2015 Accepted: 10 January 2017

Published online: 21 January 2017

\section{References}

1. Kuneš J, Vaněčková I, Mikulášková B, Behuliak M, Maletínská L, Zicha J. Epigenetics and a new look on metabolic syndrome. Physiol Res. 2015; 64(5):611.

2. Duque-Guimarães $D E$, Ozanne SE. Nutritional programming of insulin resistance: causes and consequences. Trends in Endocrinol Metab. 2013; 24(10):525-35.

3. Cani PD, Amar J, Iglesias MA, Poggi M, Knauf C, Bastelica D, et al. Metabolic endotoxemia initiates obesity and insulin resistance. Diabetes. 2007;56(7):1761-72.

4. Choi SW, Friso S. Epigenetics: a new bridge between nutrition and health. Adv Nutr. 2010;1(1):8-16.

5. Adil G, Wani SM, Masoodi FA, Gousia H. Whole-grain cereal bioactive compounds and their health benefits: a review. J Food Process Technol. 2012;3(3):146-56.

6. Zamri NDM, Imam MU, Ghafar SAA, Ismail M. Antioxidative effects of germinated brown rice-derived extracts on $\mathrm{H}_{2} \mathrm{O}_{2}$-induced oxidative stress in HepG2 cells. Evid Based Complementary Altern Med. 2014;2014:1-11.

7. Patel M, Naik S. Gamma-oryzanol from rice bran oil: a review. J Sci Ind Res. 2004;63:569-78

8. Kim JH, Kim OH, Ha YL, Kim JO. Supplementation of conjugated linoleic acid with $\gamma$-oryzanol for 12 weeks effectively reduces body fat in healthy overweight Korean women. J Food Sci Nutr. 2008;13(3):146-56.

9. Heerwagen MJ, Miller MR, Barbour LA, Friedman JE. Maternal obesity and fetal metabolic programming: a fertile epigenetic soil. Am J Physiol Regul Integr Comp Physiol. 2010;299(3):R711-22.

10. Imam MU, Ismail M. An overview on germinated brown rice and its nutragenomic implications. In: Bagachi D, Swaroop A, Bagchi M, editors. Genomics, proteomics and metabolomics in nutraceuticals and functional foods. 2nd ed. Chester, UK: John Wiley \& Sons; 2015. p. 504-17.

11. Ismail M, Abdul Hadi N, Haroun RZ, Musa SNA, Imam MU. Energy dispersive $X$-ray microanalysis of elemental distribution in raw and germinated brown rice varieties. Int J Food Prop. 2014;17(7):1449-59.

12. Musa ASN, Imam MU, Maznah I. Physicochemical properties of germinated brown rice (Oryza sativa L.) starch. Afr J Biotechnol. 2011;10(33):6281-91.

13. Imam MU, Maznah I. Nutrigenomic effects of germinated brown rice and its bioactives on hepatic gluconeogenic genes in type 2 diabetic rats and HEPG2 cells. Mol Nutr Food Res. 2013;57(3):401-11.

14. Gong H, Han YW, Sun L, Zhang Y, Zhang EY, Li Y, Zhang TM. The effects of energy intake of four different feeding patterns in rats. Exp Biol Med (Maywood). 2016;241(1):52-9.

15. D'Adamo E, Caprio S. Type 2 diabetes in youth: epidemiology and pathophysiology. Diabetes Care. 2011;34(Supplement 2):S161-5.

16. Inadera H. Developmental origins of obesity and type 2 diabetes: molecular aspects and role of chemicals. Environ Health Prev Med. 2013;18(3):185-97.

17. Yadav A, Kataria MA, Saini V, Yadav A. Role of leptin and adiponectin in insulin resistance. Clin Chim Acta. 2013;417:80-4.

18. Shin MJ, Lee JH, Jang Y, Park E, Oh J, Chung JH, et al. Insulin resistance, adipokines, and oxidative stress in nondiabetic, hypercholesterolemic patients: leptin as an 8-epi-prostaglandin F2a determinant. Metab. 2006; 55(7):918-22. 
19. Imam MU, Azmi NH, Bhanger MI, Ismail N, Ismail M. Antidiabetic properties of germinated brown rice: a systematic review. Evid Based Complement Alternat Med. 2012;2012:816501.

20. Son MJ, Rico CW, Nam SH, Kang MY. Effect of oryzanol and ferulic acid on the glucose metabolism of mice Fed with a high-Fat diet. J Food Sci. 2011; 76(1):H7-H10.

21. Friedman JM, Halaas JL. Leptin and the regulation of body weight in mammals. Nature. 1998;395(6704):763-70.

22. Liu JB, Li WJ, Fu FM, Zhang XL, Jiao L, Cao LJ, et al. Inverse correlation between serum adiponectin and 8-iso-prostaglandin F2a in newly diagnosed type 2 diabetes patients. Int J Clin Exp Med. 2015;8(4):6085-90

23. Graham TE, Yang Q, Bluher M, Hammarstedt A, Ciaraldi TP, Henry RR, et al. Retinol-binding protein 4 and insulin resistance in lean, obese and diabetic subjects. N Engl J Med. 2006;354(24):2552-63.

24. Cedar H, Bergman Y. Linking DNA methylation and histone modification: patterns and paradigms. Nat Rev Genet. 2009;10(5):295-304.

Submit your next manuscript to BioMed Central and we will help you at every step:

- We accept pre-submission inquiries

- Our selector tool helps you to find the most relevant journal

- We provide round the clock customer support

- Convenient online submission

- Thorough peer review

- Inclusion in PubMed and all major indexing services

- Maximum visibility for your research

Submit your manuscript at www.biomedcentral.com/submit
Biomed Central 